\title{
MOTION BY WEIGHTED MEAN CURVATURE IS AFFINE INVARIANT
}

\author{
JEAN E. TAYLOR
}

\begin{abstract}
Suppose curves are moving by curvature in a plane, but one embeds the plane in $R^{3}$ and looks at the plane from an angle. Then circles shrinking to a round point would appear to be ellipses shrinking to an "elliptical point," and the surface energy would appear to be anisotropic as would the mobility. The result of this paper is that if one uses the apparent surface energy and the apparent mobility, then the motion by weighted curvature with mobility in the apparent plane is the same as motion by curvature in the original plane but then viewed from the angle. This result applies not only to the isotropic case but to arbitrary surface energy functions and mobilities in the plane, to surfaces in 3-space, and (in the case that the surface energy function is twice differentiable) to the case of motion viewed through distorted lenses (i.e., diffeomorphisms) as well. This result is to be contrasted with an earlier result $[\mathrm{ST}]$ which states that for area-preserving affine transformations of the plane where the energy and mobility are NOT also transformed, motion by curvature to the power $1 / 3$ (rather than 1 ) is invariant.
\end{abstract}

\section{INTRODUCTION}

Suppose that $\Phi$ is a norm on $R^{2}$, except that it is not necessarily even. That is, $\Phi(\lambda n)=\lambda \Phi(n)$ for every $\lambda \geq 0, \Phi(n)=0$ if and only if $n=0$, and $\Phi(n+p) \leq$ $\Phi(n)+\Phi(p)$ for all $n$ and $p$ in $R^{2}$. We regard $\Phi$ as a given convex "surface" (actually curve) energy function for curves in the plane; the energy of a rectifiable oriented curve $C$ is $\int_{x \in C} \Phi\left(n_{C}(x)\right) d x$, where $n_{C}$ is the unit tangent $T_{C}$ rotated clockwise by $90^{\circ}$. The weighted (mean) curvature $\kappa_{\Phi}$ of $C$ is defined in section 2 ; it is the precise way of expressing the concept of the rate of decrease of energy of $C$ with "volume" (in $R^{2}$, area) swept out under deformations of $C$. Surface energy and weighted mean curvature are natural physical concepts, arising from the fact that for solids whose atoms are in lattices, interfaces with different normal directions relative to the lattice are quite different in structure. Various situations such as thin films are often assumed to be effectively two-dimensional.

Suppose that $M$ is also a norm that is not necessarily even, and that $C(t)$ is a family of curves in $R^{2}$ moving by weighted curvature $\kappa_{\Phi}$ with mobility $M$. That is, for each $t$ and $x \in C(t)$, the normal velocity of $C(t)$ at $x$ is $M\left(n_{C(t)}(x)\right) \kappa_{\Phi}(C(t), x)$. Motion by weighted mean curvature with mobility is likewise a standard physical concept, arising in situations such as grain growth where the materials on each side of the interface do not differ in their bulk energy. See $[\mathrm{TCH}]$ for examples and references.

Research at MSRI is supported in part by NSF grant DMS-9022140. 
Suppose $A$ is any affine map of the plane to itself; write $A(x)=L(x)+b$, where $L$ is a general linear map and $b$ a translation. For each $q \in R^{2}$, define $\Phi_{A}(q)=\Phi\left(L^{T} q\right)$ and $M_{A}(q)=M\left(L^{T} q\right)$. We show here that if $C_{A}(t)$ is the family of curves moving by weighted mean curvature $\kappa_{\Phi_{A}}$ and mobility $M_{A}$ and with $C_{A}(0)=A(C(0))$, then $A(C(t))=C_{A}(t)$ for every $t$. We also give the generalization to higher dimensions.

This result can be interpreted in the following way. Suppose you put the plane in which the curve is moving into $R^{3}$ and look at it from an angle. Then you would in fact observe motion by weighted curvature $\kappa_{\Phi_{A}}$ with mobility $M_{A}$.

The result was discovered in an attempt to understand the meaning of a result of Sapiro and Tannenbaum [ST]. They showed that for curves in the plane, motion by curvature to the one-third power, in both $C$ and $A(C)$, is invariant under areapreserving affine maps $A$. But in the anisotropic case (especially when $\Phi$ is nondifferentiable), weighted mean curvature makes no reasonable sense under affine maps unless the surface energy function is also transformed; the normal directions of $C$ can be quite different from those of $A(C)$. But once the surface energy is so transformed, then provided the mobility is also transformed, the motion which is affine invariant uses $\kappa_{\Phi}$, not $\kappa_{\Phi}^{1 / 3}$.

As surveyed and extended in [OST], the [ST] result is a special case of motions of the form where the time derivative in the normal direction is equal to the second spatial derivative in a metric which is invariant under some group action. The interpretation of this paper in those terms is that one has a metric defined only on $C$, with distance along $C$ being given by $\Phi$ and normal to $C$ being given by $M$. When one maps $C$, $\Phi$, and $M$ by $A$, one changes the metric in such a way that these "distances" remain invariant.

The original purpose of the Sapiro and Tannenbaum work, however, was with regard to image processing: motion by curvature is useful to remove noise. In that case, there is no physical Wulff shape, and the meaning of curvature as the rate of decrease of length with area swept out is irrelevant. Conversely, it is not clear whether the result of this paper has any useful implications for image processing.

A final note concerns the importance of the mobility. That there should be a mobility factor is evident from considering units: the units of velocity are distance over time, and the units of curvature are one over distance. In our case, even if mobility is initially 1 on each unit normal vector and so "invisible," it must be transformed and used in the $C_{A}$ motion, or the original motion is not preserved under affine maps. Mobility shows up naturally in other places as well. For example, in order for Wulff shapes to shrink homothetically under motion by weighted mean curvature with mobility, $M$ must be proportional to $\Phi$. And in the anisotropic AllenCahn equation, the diffuse interface approach to motion by weighted mean curvature, the sharp interface limit naturally produces an extra factor in $M$ of $\Phi$ in addition to whatever diffuse mobility is initially put into the Allen-Cahn equation.

\section{Definitions}

For further details and references concerning this section, see [T1] [T2]. 
2.1 The Wulff shape of $\Phi$ is the convex body $W_{\Phi}=\{x: x \cdot p \leq \Phi(p) \quad \forall p\}$; it is the equilibrium crystal shape. If $\Phi$ is nondifferentiable in direction $n$, then $W_{\Phi}$ has a facet with normal $n$; let $\Lambda_{\Phi}(n)$ be its length.

2.2.1 If $C$ is a twice differentiable curve at $x$ in $C$ and $\Phi$ is twice differentiable at $n_{C}(x)$, then $\kappa_{\Phi}(C, x)=-\left.T_{C}(x) \cdot \frac{d}{d s} \nabla \Phi\right|_{n_{C}(x)}$ where $\frac{d}{d s}$ denotes differentiation with respect to arc length along $C$. That is, it is minus the "surface" divergence of $\nabla \Phi$. One computes that if $C$ is the graph of $x_{2}=\frac{1}{2 r} x_{1}+o\left(x_{1}^{2}\right)$ near $(0,0)$, with oriented tangent $(1,0)$ at $(0,0)$, then $\kappa_{\Phi}(C,(0,0))=-\left.\frac{1}{r} \frac{\partial^{2} \Phi}{\partial p_{1}^{2}}\right|_{p=(0,-1)}$, the curvature times a weight.

2.2.2 If $C$ around $x$ is a line segment, of length $\ell$, and $\Phi$ is nondifferentiable at $n_{C}(x)$, then $\kappa_{\Phi}(C, x)=-\sigma \Lambda_{\Phi}\left(n_{C}(x)\right) / \ell$, where $\sigma$ is 1 if $C$ is locally convex at each end of the line segment, $\sigma$ is -1 if $C$ is locally concave at each end, and $\sigma=0$ if $C$ is convex near one end and is concave near the other.

2.2.3 The definition of $\kappa_{\Phi}$ in the smooth case comes from the fact that $\Phi$-firstvariation linear operator on vector fields $g$ is representable as

$$
-\int_{x \in C} g(x) \cdot n_{C}(x) \kappa_{\Phi}(C, x) d x
$$

The non-differentiable case comes from using non-local deformations. If $n_{C}(x)$ does not exist, then either there is an energy minimizing tangent cone to $C$ at $x$ or else the weighted mean curvature there is infinite.

2.3 Note that the unit normal $n_{C}(x)$ is oriented here as the exterior normal to a region in the plane locally bounded by $C$, as a special case of the unit oriented normal $n_{S}(x)$ to a surface $S$ bounding a region in $R^{d}$. The curvature vector field $\vec{\kappa}$ points in the direction of maximum decrease of length under variations, and the sign of the curvature here is such that $\vec{\kappa}=\kappa n_{C}$. The curvature of the boundary of a convex body is thus negative.

\section{Statements And Proofs}

Lemma 3.1. Given $v$ in $R^{2}$, if $q$ is normal to $L(v)$, then $L^{T} q$ is normal to $v$.

Proof. $q \cdot L(v)=L^{T} q \cdot v$.

Lemma 3.2. $W_{\Phi_{A}}=L\left(W_{\Phi}\right)$.

Proof. $W_{\Phi_{A}}=\left\{x: x \cdot q \leq \Phi_{A}(q) \quad \forall q\right\}=\left\{x: x \cdot q \leq \Phi\left(L^{T} q\right) \quad \forall q\right\}=\{x:$ $\left.x \cdot\left(L^{T}\right)^{-1} p \leq \Phi(p) \quad \forall p\right\}=\left\{x: L^{-1} x \cdot p \leq \Phi(p) \quad \forall p\right\}=\{L y: y \cdot p \leq \Phi(p) \quad \forall p\}=$ $L\left(W_{\Phi}\right)$.

Lemma 3.3. $\kappa_{\Phi_{A}}(A(C), A(x))=\kappa_{\Phi}(C, x)$.

Proof. Consider first the case that $\Phi$ is not differentiable at $n=n_{C}(x)$, so that the boundary of $W_{\Phi}$ has a facet (line segment) $W$ with normal $n$. Suppose $S$ is the maximal line segment in $C$ through $x$. By lemma 3.2, the length of $L(W)$ is $\Lambda_{\Phi_{A}}(q)$ for some $q$, and by lemma $3.1, q=\left(L^{T}\right)^{-1} n /\left|\left(L^{T}\right)^{-1} n\right|$, which is normal to $A(S)$. Since $S$ is parallel to $W$, the ratio of lengths of $L(W)$ and $A(S)$ is that of $W$ and $S$. Thus $\kappa_{\Phi}(C, x)=\kappa_{\Phi_{A}}(A(C), A(x))$. 
If $\Phi$ is differentiable at $n$, the lemma can be proved by approximation by nondifferentiable $\Phi$. It can also be obtained by the characterization of the weighted mean curvature as the rate of decrease in surface energy with the volume (with $d=2$, area) swept out, with a proof as in section 4 of this paper. Or it can be obtained as an exercise in direct computation, as follows. Assume without loss of generality that $n=(0,-1)$ and write $C$ locally as a graph $x_{2}=\frac{1}{2 r} x_{1}^{2}+o\left(x_{1}^{2}\right)$. One computes

$$
\begin{gathered}
-\kappa_{\Phi_{A}}(A(C), A(0,0))=\left.\left(\left.\operatorname{div}_{A(C)} \nabla \Phi A\right|_{p=\left(x_{1}, x_{1}^{2} /(2 r)\right)}\right)\right|_{x_{1}=0} \\
=\left.\frac{L(0,-1)}{|L(0,-1)|} \cdot\left|\frac{d A\left(x_{1}, x_{1}^{2} /(2 r)\right)}{d x_{1}}\right|^{-1}\left(\left.\frac{d}{d x_{1}}\left(\frac{d \Phi\left(L^{T} q\right)}{d q_{1}}, \frac{d \Phi\left(L^{T} q\right)}{d q_{2}}\right)\right|_{q=n_{A(C)}\left(A\left(x_{1}, x_{1}^{2} /(2 r)\right)\right)}\right)\right|_{x_{1}=0} \\
=\left.\frac{1}{r} \frac{\partial^{2} \Phi}{\partial p_{1}^{2}}\right|_{p=(0,-1)},
\end{gathered}
$$

remembering that $\frac{\partial^{2} \Phi}{\partial p_{1} \partial p_{2}}(0,-1)=0$ as a result of $p \cdot \nabla \Phi(p)=\Phi(p)$ for all $p$, which in turn follows from $\Phi(p)=|p| \Phi(p /|p|)$ for all nonzero $p$.

Theorem 3.4. If $C(t)$ is a family of curves for $t \in\left[0, t_{0}\right]$, with normal velocity $M \kappa_{\Phi}$ and if $C_{A}(t)$ is a family of curves with normal velocity $M_{A} \kappa_{\Phi_{A}}$ and $C_{A}(0)=A(C(0))$, then $A(C(t))=C_{A}(t)$ for all $t \in\left[0, t_{0}\right]$.

Proof. By hypothesis, $A(C(t))=C_{A}(t)$ when $t=0$; suppose it remains true up until time $t \in\left[0, t_{0}\right)$. If $q$ is the unit normal to $C_{A}(t)=A(C(t))$ at $A(x)$ for some $x$, then $L^{T} q /\left|L^{T} q\right|$ is the unit normal to $C(t)$ at $x$. If $d s$ is the incremental motion of $x$ in the direction $n_{C(t)}(x)$, then $q \cdot L\left(L^{T} q /\left|L^{T} q\right|\right) d s=\left|L^{T} q\right| d s$ is $A(d s)$, the normal component of the motion of $A(C(t))$ at $A(x)$. We now plug in $d s=$ $M\left(L^{T} q /\left|L^{T} q\right|\right)\left(-\sigma \frac{\Lambda}{\ell}\right) d t$ to obtain $A(d s)=M\left(L^{T} q\right)\left(-\sigma \frac{\Lambda}{\ell}\right) d t$. But this is precisely the incremental normal motion of $C_{A}(t)$, given Lemma 3 and the definition $M_{A}(q)=$ $M\left(L^{T} q\right)$.

Singularities can be present initially or can develop if $\Phi$ is not even. In fact, one might be dealing with two-dimensional grain growth where there are many triple junctions at all times. Whatever way one handles such singular points for $\Phi$ (see [T2] for example), they should then by definition be handled in the same way for $\Phi_{A}$. Since $A$ maps any such singular points in $C(t)$ to similar singular points in $C_{A}(t)$, the proof above carries over.

\section{HighER DIMENSIONS}

Suppose $S$ is a $\left(d\right.$-1)-dimensional oriented rectifiable surface in $R^{d}, \Phi$ and $M$ are norms on $R^{d}$, and $A$ is an affine map on $R^{d}$, with $A(x)=L(x)+b$. Again define $\Phi_{A}(q)=\Phi\left(L^{T} q\right)$ and $M_{A}(q)=M\left(L^{T} q\right)$. The critical computation is

$$
\begin{gathered}
\Phi_{A}(A(S))=\int_{y \in A(S)} \Phi_{A}\left(n_{A(S)}(y)\right) d y \\
=\int_{y \in A(S)} \frac{\Phi\left(n_{S}\left(A^{-1} y\right)\right)}{\left|\left(L^{-1}\right)^{T} n_{S}\left(A^{-1} y\right)\right|} d y
\end{gathered}
$$




$$
\begin{gathered}
=\int_{x \in S} \frac{\Phi\left(n_{S}(x)\right)}{\left|\left(L^{-1}\right)^{T} n_{S}(x)\right|} J_{2} L\left(n_{S}(x)\right) d x \\
=\int_{x \in S} \Phi\left(n_{S}(x)\right)|\operatorname{det} L| d x .
\end{gathered}
$$

Since weighted mean curvature is the rate of decrease of surface energy with volume, and the volume changes by a factor of $|\operatorname{det} L|$ under mapping by $A$, once again the weighted mean curvature of $S$ at $x$, with respect to surface energy $\Phi$, is the same as the weighted mean curvature of $A(S)$ at $A(x)$, with respect to surface energy $\Phi_{A}$. The same proof as before shows that once the mobility is also transformed, motion by weighted mean curvature with mobility is affine invariant (i.e., $A(C(t))=C_{A}(t)$ ).

If $\Phi$ is differentiable, then one can extend the results further to the case of nonconstant-coefficient $\Phi$ and to diffeomorphisms $f: R^{d} \rightarrow R^{d}$ rather than just affine maps $A$, since the preceding computation of surface energy is entirely local. Given $\Phi$ depending on $x$ and $p$, one defines $\Phi_{f}$ by $\Phi_{f}(y, q)=\Phi\left(x,\left(D f_{x}\right)^{T} q\right)$ where $x=f^{-1}(y)$. Here

$$
\int_{y \in f(S)} \Phi_{f}\left(n_{f(S)}(y)\right) d y=\int_{x \in S} \Phi\left(x, n_{S}(x)\right)\left|\operatorname{det} D f_{x}\right| d x .
$$

Again, weighted mean curvature stays the same, the mobility changes, and motion by weighted mean curvature with mobility is affine invariant.

This final result has the following interpretation: not only can you look at motion by weighted mean curvature with mobility at an angle, but you can look at it through a distorted lens. As long as you use your locally-measured $\Phi_{f}$ and $M_{f}$, you will observe motion by weighted mean curvature with mobility, provided the original motion is by the actual weighted mean curvature with mobility.

\section{ACKNOWLEDGEMENTS}

This work was partially supported by a grant from the National Science Foundation. The author is also grateful for the hospitality of MSRI, where this paper was completed, and to Karen Almgren for a careful reading of the paper.

\section{REFERENCES}

[OST] Peter J. Olver, Guillermo Sapiro, and Allen Tannenbaum, Invariant geometric evolution of surfaces and volumetric smoothing, SIAM J. Appl. Math. 57 (1997), 176-194.

[ST] Guillermo Sapiro and Allen Tannenbaum, On affine plane curvature evolution, J. Functional Anal. 119 (1994), 79-120.

[T1 Jean E. Taylor, Mean curvature and weighted mean curvature, Acta metallurgica et materialia 40 (1992), 1475-1485.

[T2] Jean E. Taylor, Motion of curves by crystalline curvature, including triple junctions and boundary points, Differential Geometry, Proceedings of Symposia in Pure Math. 51 (part 1) (1993), 417-438. 
[TCH] Jean E. Taylor, John W. Cahn and Carol A. Handwerker, Geometric Models of Crystal Growth, Acta Metall. Mater. 40 (1992), 1443-1474.

Mathematics Department, Rutgers University, Piscataway, NJ 08855

E-mail address: taylor@math.rutgers.edu 